\title{
FUZZY IMAGE SEGMENTATION USING VALIDITY INDEXES CORRELATION
}

\author{
Daniel Fernando Tello Gamarra ${ }^{1}$ \\ ${ }^{1}$ Department of Control Engineering and Automation, Universidade Federal de Santa \\ Maria (UFSM), Santa Maria, Brazil
}

\begin{abstract}
This paper introduces an algorithm for image segmentation using a clustering technique; the technique is based on the fuzzy c means algorithm (FCM) that is executed iteratively with different number of clusters. Furthermore, simultaneously five validity indexes are calculated and their information is correlated to determine the optimal number of clusters in order to segment an image, results and simulations are shown in the paper.
\end{abstract}

\section{KEYWORDS}

Image Segmentation, Validity Indexes, Fuzzy C Means, Indexes Correlation.

\section{INTRODUCTION}

Image processing is used in applications for different fields like medicine, industry or robotics ([1] [2] [3] [4] [5]). Segmentation is the first step in image processing and is the supports for the execution of other tasks such as refinement or features identification as referred in [6]. Segmentation is a low-level operation with the objective of dividing homogeneous or heterogeneous regions. The homogeneous regions with borders belong to objects or part of them in the images. The approach for segmentation followed in this paper applies Fuzzy logic, there are some previous efforts executed following this approach, for instance, Lopes in [7] uses an automatic threshold method based on a fuzziness measure; Cheng in [8] uses principles of fuzzy c-partition and the maximum entropy to select threshold values for gray-level images.

Other topic that explores this work is the use of validity indexes. Previously, Gamarra in [9] applied validity indexes to find the optimal number of clusters. Besides, some interesting works have explored the application of validity indexes for image segmentation. Bensaid in [10] proposed a new validity index, the partition index (SC), and introduced a new clustering algorithm, the validity-guided (re) clustering (VGC), in Bensaid's work the validity index is not used to find the optimal number of clusters, that is already known; the validity index is used to improve the quality of partitions generated by a clustering algorithm. The VGC was applied exclusively for Magnetic Resonance Images (MRI) images. In addition, the work developed for Velthuizen in [11] applies the VGC algorithm to MRI images for brain tumor segmentation.

This work will explore the application of different validity indexes, which will have their information correlated in order to find an adequate number of clusters for an image segmentation task. The remainder of the paper is as follows. the second section explains the different algorithms used in the paper; the third section presents the proposed algorithm designed for the image segmentation; in the fourth section the experimental platform setup is detailed; in the fifth section the experimental results are described; finally, conclusions are drawn in the last section. 
International Journal of Computer Science \& Information Technology (IJCSIT) Vol 7, No 3, June 2015

\section{THEORETICAL BACKGROUND}

\subsection{Fuzzy C Means Algorithm (FCM)}

As stated in [12], fuzzy $\mathrm{C}$ means is very simple; unsupervised and convergent algorithm. The algorithm description uses equations and terminology written in [13] and resumed in [9]. Given the data set $X$, chose the number of clusters $c$, between $1<c<N, N$ is the number of training data objects, the weighting exponent $m>1$, the termination tolerance $\varepsilon>0$ and the norm-inducing matrix $A$, if $A=I$ gives the standard Euclidean norm as stated in [14].

The matrix $U$ is the fuzzy partition matrix and represents the membership of the data samples in the clusters. The weight $\mu_{i k}$ represents the degree of membership of an object in a cluster, and is contained in the kth column of $U$. An object belongs in some degree to the different clusters, and each cluster has a centroid $v_{i}$, if the number of iterations is $l$, the first step of the algorithm is to compute the cluster centroids:

$$
v_{i}^{(l)}=\frac{\sum_{k=1}^{N}\left(\mu_{i k}^{l-1}\right)^{m} x_{k}}{\sum_{k=1}^{N}\left(\mu_{i k}^{l-1}\right)^{m}}, 1 \leq i \leq c
$$

The second step computes the distances:

$$
\begin{aligned}
d_{i k A}^{2} & =\left(x_{k}-v_{i}^{(l)}\right)^{T} A\left(x_{k}-v_{i}^{(l)}\right), \\
1 & \leq i \leq c, 1 \leq k \leq N
\end{aligned}
$$

The third step updates the partition matrix elements:

$$
\text { If } \begin{aligned}
d_{i k A}>0 \text { for } 1 \leq i \leq c, 1 \leq k \leq N, & 1 \\
\mu_{i k}^{(l)}= & \frac{1}{\sum_{j=1}^{c}\left(d_{i k A} / d_{j k A}\right)^{2 /(m-1)}}
\end{aligned}
$$

Otherwise $\mu_{i k}^{(l)}=0$ if $d_{i k A}=0$ and $\mu_{i k}^{(l)} \in[0,1]$ with $\sum_{i=1}^{c} \mu_{i k}^{l}=1$.

The algorithm lasts a fixed number of iterations or until is reached the condition $\left\|U^{(l)}-U^{(l-1)}\right\|<\varepsilon$

\subsection{Validity Indexes in Fuzzy Clustering}

The cluster validity as stated in [15] can be used to search for the optimal number of clusters if the number of clusters is not known. The formulation followed in this subsection took as a reference the work developed in [15]. The validity indexes used for this paper are the following and derived from [16]:

The Partition Coefficient (PC) is defined as follows:

$$
P C=\frac{1}{n} \sum_{i=1}^{c} \sum_{j=1}^{n} u_{i j}^{2}
$$


The Partition Entropy (PE):

$$
P E=\frac{1}{n} \sum_{i=1}^{c} \sum_{j=1}^{n} u_{i j}^{2} \log u_{i j}
$$

The partition index (SC) given by the equation:

$$
S C=\sum_{i=1}^{c} \frac{\sum_{j=1}^{N}\left(u_{i j}\right)^{m}\left\|x_{j}-v_{i}\right\|^{2}}{N_{i} \sum_{k=1}^{c}\left(u_{i j}\right)^{m}\left\|x_{j}-v_{i}\right\|^{2}}
$$

The Separation index (S) on the contrary of SC uses a minimum-distance separation for validity partition [10]:

$$
S=\frac{\sum_{i=1}^{c} \sum_{j=1}^{N}\left(u_{i j}\right)^{m}\left\|x_{j}-v_{i}\right\|^{2}}{N \min _{i, k}\left\|v_{k}-v_{i}\right\|^{2}}
$$

The Xie and Beni's index defined in [17] as follows:

$$
X B=\frac{\sum_{i=1}^{c} \sum_{j=1}^{n} u_{i j}^{m}\left\|x_{j}-v_{j}\right\|^{2}}{N \min _{i \neq j}\left\|x_{j}-v_{j}\right\|^{2}}
$$

\section{Proposed Algorithm}

Figure 1 depicts the algorithm flowchart, the algorithm will start using a median filter, as the first step; the next step will be the application of a clustering technique for image segmentation based in the Fuzzy C Means algorithm. One of the parameters that are necessary to specify for the FCM is the number of clusters in which the image data will be divided, different cluster numbers were tested for the algorithm, beginning with 2 clusters until a predefined number of clusters is achieved (it was chosen 14 for the experiments in this paper). The different validity indexes that were defined in the paper will evaluate the partitions created for the Fuzzy C Means algorithm in every run of the algorithm, based on the analysis and the correlation of information provided for the validity indexes an optimal number of clusters will be selected.

\section{EXPerimental Platform Setup}

\subsection{The Matlab Image Processing Toolbox}

For the experiments developed in this paper the matlab image processing toolbox will be employed, this toolbox has a set of routines that let us handle a lot of computational vision problems.

\subsection{The Fuzzy Clustering Toolbox}

For the cluster validity was used the Fuzzy Clustering and Data Analysis Toolbox [18]. The referred toolbox is a collection of Matlab functions with the purpose of dividing a given data set into clusters. 


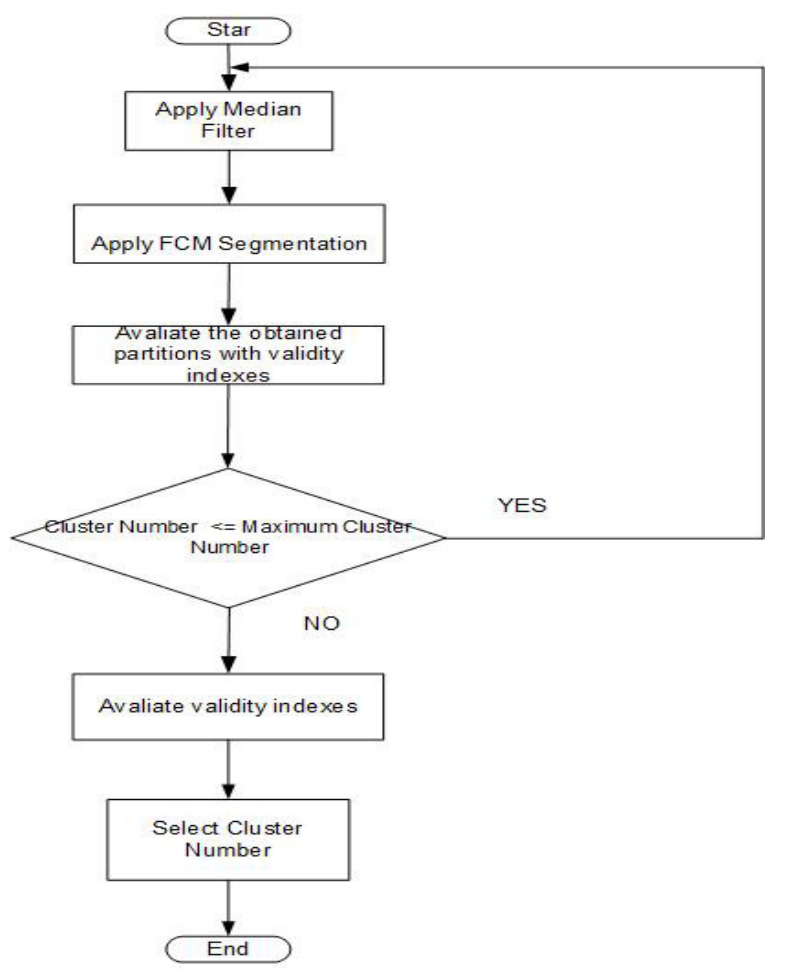

Figure 1. Proposed Algorithm Flowchart

\section{EXPERIMENTAL RESULTS}

The different experiments were done with the proposed algorithm in this paper. The parameters used for the clustering algorithm are: number of clusters $=4$, maximum number of iterations $=$ $100(l)$, partition matrix exponent $=2(\mathrm{~m})$, amount of minimal improvement $=1 \mathrm{e}-6(\varepsilon)$.

Figure 2 shows the classic figure of the cameraman, that is clustered using the method proposed on the paper; figure 3 depicts the results obtained applying the validity indexes to the figure, the partition coefficient (PC) has a monotonic decreasing starting in cluster number 4 , also the classification entropy (CE) has a monotonic increasing starting in cluster 4 . Figure 4 shows that the partition index $(\mathrm{SC})$ and the separation index $(\mathrm{S})$ decreases slowly from $\mathrm{c}=4$. Also the Xie and Beni index reaches its local minimum in a region between $\mathrm{c}=3 \mathrm{e} \mathrm{c}=4$. After an evaluation the information deliver from SC and S are more useful in comparison with the other indexes with the same $\mathrm{c}$, it was chosen 4 as the optimal number of clusters.

In Table 1 appears the different values obtained for every cluster validity index adopted and its variation through the different number of clusters tested. $\mathrm{c}$ is the clusters number and PC, CE, SC, $\mathrm{S}$ and $\mathrm{XB}$ the initials of validity indexes. 
International Journal of Computer Science \& Information Technology (IJCSIT) Vol 7, No 3, June 2015

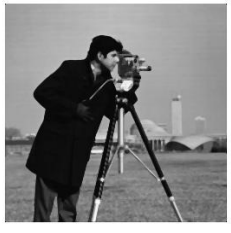

(a)

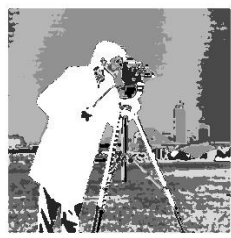

(c)

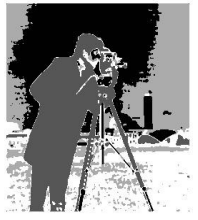

(b)

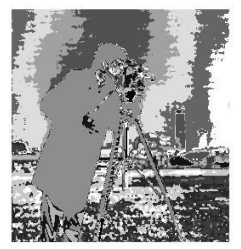

(d)

Figure. 2. Cameraman clustered image with FCM: a) original image; (b) 4 clusters; (c) 8 clusters; (d) 14 clusters
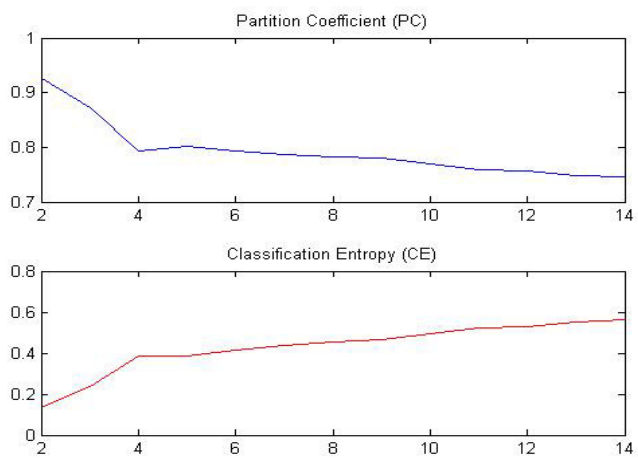

Figure 3. Values of Partition Coefficient and Classification Entropy with the clusters number for the cameraman image.
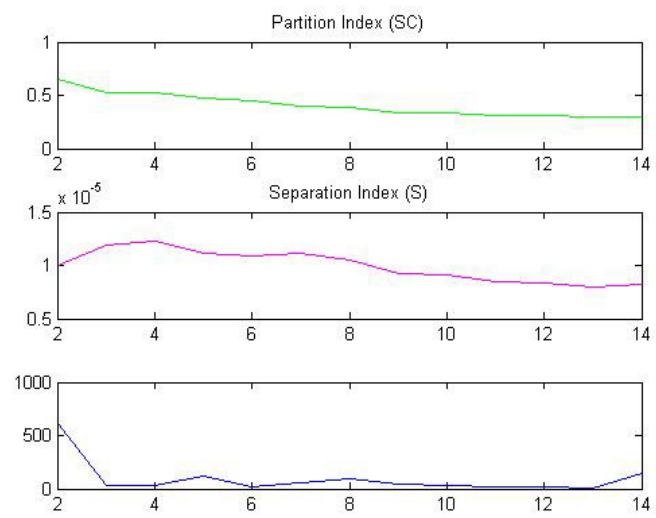

Figure 4 Values of Partition Index, Separation Index, and Xie and Beni's Index with the clusters number for the cameraman image. 
International Journal of Computer Science \& Information Technology (IJCSIT) Vol 7, No 3, June 2015

Table 1 The Numerical Values of Validity Measures ( Cameraman)

\begin{tabular}{cccccccl}
\hline \hline $\mathrm{C}$ & 2 & 3 & 4 & 5 & 6 & 7 & 8 \\
\hline $\mathrm{PC}$ & 0.9271 & 0.8731 & 0.7926 & 0.8020 & 0.7939 & 0.7872 & 0.7832 \\
$\mathrm{CE}$ & 0.1348 & 0.2351 & 0.3839 & 0.3841 & 0.4114 & 0.4372 & 0.4557 \\
$\mathrm{SC}$ & 0.6546 & 0.5240 & 0.5314 & 0.4808 & 0.4544 & 0.4024 & 0.3809 \\
$\mathrm{~S}$ & 0.0999 & 0.1195 & 0.1228 & 0.1109 & 0.1085 & 0.1111 & 0.1053 \\
$10-4$ & & & & & & & \\
& & & & & & & \\
XB & 622.39 & 32.7227 & 29.3341 & 117.1989 & 17.7296 & 61.406 & 97.276 \\
\hline $\mathrm{C}$ & 9 & 10 & 11 & 12 & 13 & 14 & \\
\hline PC & 0.7808 & 0.7686 & 0.7586 & 0.7575 & 0.7480 & 0.7470 & \\
CE & 0.4664 & 0.4968 & 0.5214 & 0.5295 & 0.5538 & 0.5626 & \\
SC & 0.3376 & 0.3331 & 0.3111 & 0.3126 & 0.2968 & 0.2925 & \\
S & 0.0927 & 0.0911 & 0.0843 & 0.0839 & 0.0800 & 0.0819 & \\
XB & 50.509 & 29.6524 & 23.3215 & 15.9614 & 9.5482 & 146.39 & \\
\hline \hline
\end{tabular}

Figure 5 shows the classic figure of lena, that is clustered using the method proposed on the paper; figure 6 illustrates the results obtained applying the validity indexes to the figure, the partition coefficient (PC) has a monotonic decreasing starting in cluster number 3 , also the classification entropy (CE) has a monotonic increasing starting in cluster 3. Figure 7 shows that the partition index (SC) and the separation index (S) decreases slowly from $\mathrm{c}=3$. Also the Xie and Beni index reaches its local minimum at $\mathrm{c}=3$. After an evaluation of the information deliver for $\mathrm{SC}$ and $\mathrm{S}$ for this case is more useful in comparison with the other indexes with the same $\mathrm{c}$, it was chosen 3 as the optimal number of clusters.

In table 2 are shown the different values obtained for every cluster validity indexes adopted and its variation through the different number of clusters tested.

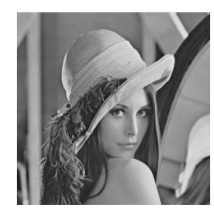

(a)

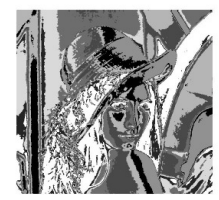

(c)

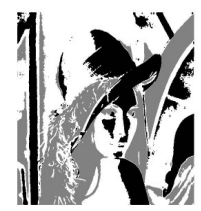

(b)

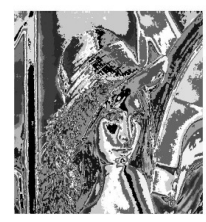

(d)

Figure 5. Lena clustered with FCM: a) original image; (b) 3 clusters; (c) 8 clusters; (d) 14 clusters 
International Journal of Computer Science \& Information Technology (IJCSIT) Vol 7, No 3, June 2015
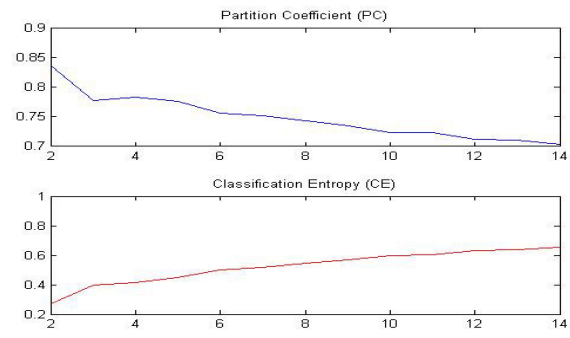

Figure. 6. Values of Partition Coefficient and Classification Entropy with the clusters number for lena image.
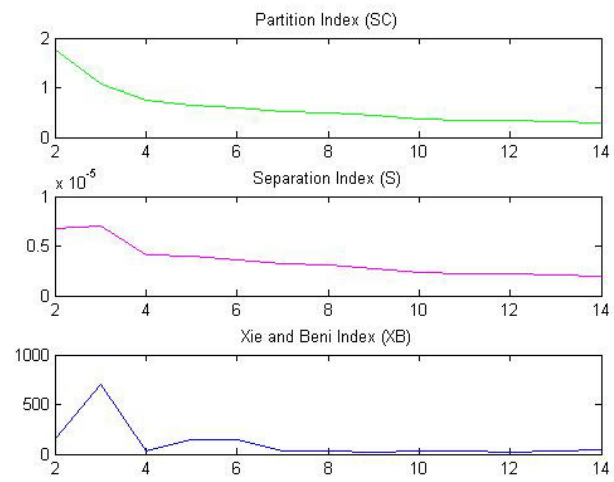

Figure 7. Values of Partition Index, Separation Index, and Xie and Beni's Index with the clusters number for lena image.

Table 2 The Numerical Values of Validity Measures (Lena)

\begin{tabular}{cccccccl}
\hline \hline $\mathrm{C}$ & 2 & 3 & 4 & 5 & 6 & 7 & 8 \\
\hline $\mathrm{PC}$ & 0.8373 & 0.7781 & 0.7834 & 0.7761 & 0.7568 & 0.7526 & 0.7434 \\
$\mathrm{CE}$ & 0.2719 & 0.3975 & 0.4169 & 0.44841 & 0.5014 & 0.5189 & 0.5466 \\
$\mathrm{SC}$ & 1.7675 & 1.0729 & 0.7387 & 0.6438 & 0.6065 & 0.5095 & 0.4912 \\
$\mathrm{~S}$ & 0.6742 & 0.7052 & 0.4121 & 0.4028 & 0.3669 & 0.3254 & 0.3066 \\
$(10-$ & & & & & & & \\
$5)$ & & & & & & & \\
$\mathrm{XB}$ & 154.26 & 700.47 & 33.967 & 147.051 & 142.2324 & 29.471 & 29.123 \\
\hline $\mathrm{C}$ & 9 & 10 & 11 & 12 & 13 & 14 & \\
\hline $\mathrm{PC}$ & 0.7348 & 0.7237 & 0.7233 & 0.7118 & 0.7114 & 0.7039 & \\
$\mathrm{CE}$ & 0.5709 & 0.5953 & 0.6017 & 0.6302 & 0.6365 & 0.6553 & \\
$\mathrm{SC}$ & 0.4380 & 0.3683 & 0.3538 & 03492 & 0.3526 & 0.2977 & \\
$\mathrm{~S}$ & 0.2767 & 0.2351 & 0.2242 & 0.2197 & 0.2064 & 0.1903 & \\
$\mathrm{XB}$ & 15.935 & 30.769 & 25.815 & 24.7709 & 31.8063 & 38.095 & \\
\hline \hline
\end{tabular}

Figure 8 shows the lama image from the Ground Truth database ([19]), that is clustered using the method proposed on this paper. Figure 9 shows the results obtained applying the validity index to the figure, the partition coefficient (PC) has a monotonic decreasing starting with cluster number 4 , also the classification entropy (CE) has a monotonic increasing starting in cluster 4 . Figure 8 shows that the partition index (SC) and the separation index (S) are fixed constant at $\mathrm{c}=4$. Also the Xie and Beni index reaches its local minimum at $\mathrm{c}=4$. Considering that $\mathrm{SC}$ and $\mathrm{S}$ are more 
useful, when comparing different clustering methods with the same c, it was chosen 4 as the optimal number of clusters. Evidently, this figure contains four clusters and the algorithm proves this fact.

In table 3 are shown the different values obtained for every cluster validity indexes adopted and its variation through the different number of clusters tested.

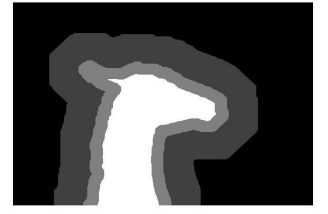

(a)

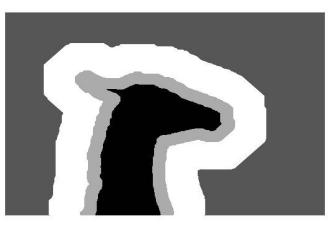

(c)

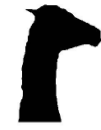

(b)

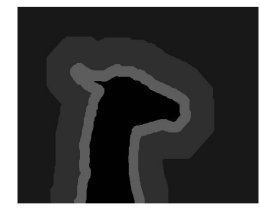

(d)

Figure 8. Llama image clustered with FCM: a) original image; (b) 2 clusters; (c) 4 clusters; (d) 14 clusters.
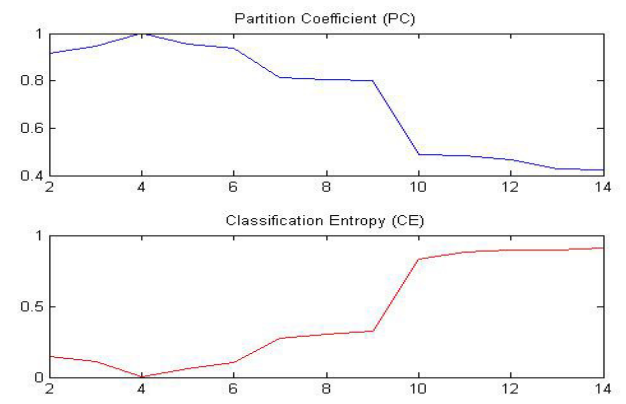

Figure 9. Values of Partition Coefficient and Classification Entropy with the clusters number for lama image.
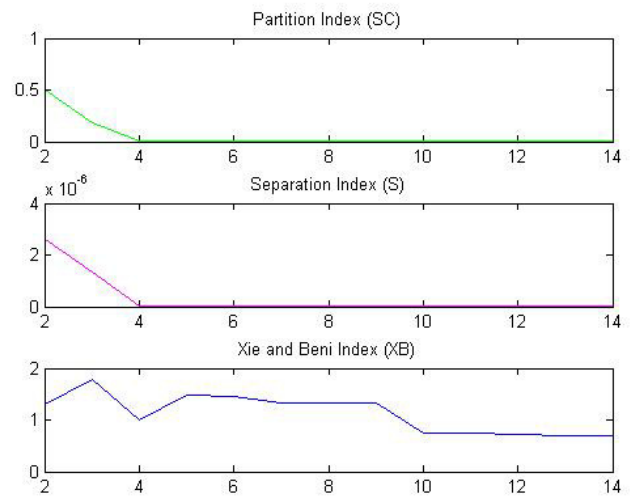

Figure 10. Values of Partition Index, Separation Index, and Xie and Beni's Index with the clusters number for lama image. 
International Journal of Computer Science \& Information Technology (IJCSIT) Vol 7, No 3, June 2015

Table 3 The Numerical Values of Validity Measures (Llama Image)

\begin{tabular}{cccccccl}
\hline \hline $\mathrm{C}$ & 2 & 3 & 4 & 5 & 6 & 7 & 8 \\
\hline $\mathrm{PC}$ & 0.9171 & 0.9455 & 1,000 & 0.9543 & 0.9391 & 0.8130 & 0.8054 \\
$\mathrm{CE}$ & 0.1494 & 0.1088 & 0.000 & 0.0633 & 0.1003 & 0.2752 & 0.3015 \\
$\mathrm{SC}$ & 0.5005 & 0.1874 & 0 & 0 & 0 & 0. & 0. \\
$\mathrm{~S}$ & 0.2630 & 0.1334 & 0 & 0 & 0 & 0 & 0 \\
$(10-$ & & & & & & & \\
$5)$ & & & & & & & \\
$\mathrm{XB}$ & 1.2897 & 1.7839 & 1 & 1.4793 & 1.4642 & 1.3380 & 1.3304 \\
\hline $\mathrm{C}$ & 9 & 10 & 11 & 12 & 13 & 14 & \\
\hline $\mathrm{PC}$ & 0.8008 & 0.4900 & 0.4816 & 0.4672 & 0.4275 & 0.4253 & \\
$\mathrm{CE}$ & 0.3219 & 0.8333 & 0.8793 & 0.8966 & 0.8958 & 0.9099 & \\
$\mathrm{SC}$ & 0 & 0 & 0 & 0 & 0 & 0 & \\
$\mathrm{~S}$ & 0 & 0 & 0 & 0 & 0 & 0 & \\
$\mathrm{XB}$ & 1.3258 & 0.7528 & 0.7444 & 0.7297 & 0.6900 & 0.6878 & \\
\hline \hline
\end{tabular}

Figure 11 shows the cell image from the matlab image processing toolbox, that is clustered using the method proposed on this paper; figure 12 depicts the results obtained applying the validity index to the figure, the partition coefficient (PC) has a monotonic decreasing starting in cluster number 3, also the classification entropy (CE) has a monotonic increasing starting in cluster 3. From figure 13, it is possible to observe that the partition index (SC) and the separation index (S) hardly decreases at $\mathrm{c}=3$. Also the Xie and Beni index reaches its local minima at $\mathrm{c}=3$. After an evaluation of the information deliver for $\mathrm{SC}$ and $\mathrm{S}$ are for this case more useful in comparison with the other indexes with the same c, it was chosen 3 as the optimal number of clusters.

In table 4 are shown the different values obtained for every cluster validity indexes adopted and its variation through the different number of clusters tested.

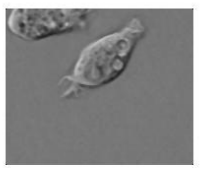

(a)

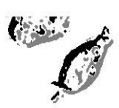

(c)

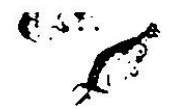

(b)

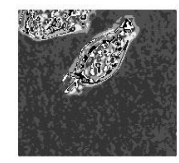

(d)

Figure 11. Cell image clustered with FCM: a) original image; (b) 2 clusters; (c) 3 clusters; (d) 12 clusters 
International Journal of Computer Science \& Information Technology (IJCSIT) Vol 7, No 3, June 2015
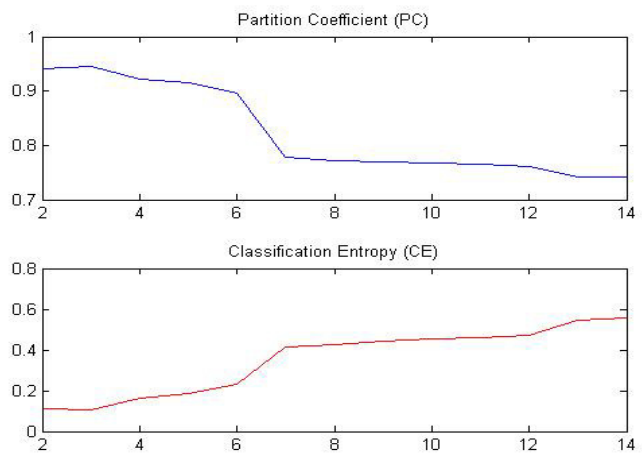

Figure 12. Values of Partition Coefficient and Classification Entropy with the clusters number for the cell image.
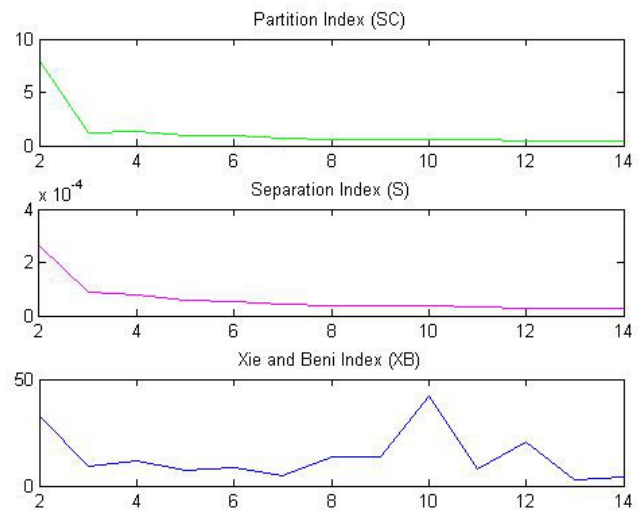

Figure 13. Values of Partition Index, Separation Index, and Xie and Beni's Index with the clusters number for the cell image.

Table 4 The Numerical Values of Validity Measures (Cell Image)

\begin{tabular}{cccccccc}
\hline \hline $\mathrm{C}$ & 2 & 3 & 4 & 5 & 6 & 7 & 8 \\
\hline $\mathrm{PC}$ & 0.9416 & 0.9449 & 0.9223 & 0.9154 & 0.8951 & 0.7787 & 0.7715 \\
$\mathrm{CE}$ & 0.1090 & 0.1085 & 0.1646 & 0.1841 & 0.2342 & 0.4140 & 0.4272 \\
$\mathrm{SC}$ & 8.0434 & 1.1791 & 1.3021 & 0.8921 & 0.8948 & 0.6984 & 0.5633 \\
$\mathrm{~S}$ & 0.2649 & 0.0881 & 0.0797 & 0.0584 & 0.0546 & 0.0451 & 0.0374 \\
$(10-3)$ & & & & & & \\
$\mathrm{XB}$ & 32.632 & 8.9606 & 11.9188 & 6.99 & 8.6761 & 4.7263 \\
$\mathrm{C}$ & 9 & 10 & 11 & 12 & 13 & 14.4435 \\
$\mathrm{PC}$ & 0.7703 & 0.7670 & 0.7658 & 0.7621 & 0.7424 & 0.7410 \\
$\mathrm{CE}$ & 0.4440 & 0.4566 & 0.4623 & 0.4694 & 0.5468 & 0.5573 \\
$\mathrm{SC}$ & 0.5812 & 0.5901 & 0.5073 & 0.4321 & 0.3905 & 0.4032 \\
$\mathrm{~S}$ & 0.0369 & 0.0368 & 0.0322 & 0.0280 & 0.0257 & 0.0258 \\
$\mathrm{XB}$ & 13.569 & 41.9482 & 7.9208 & 20.5686 & 2.667 & 4.2716 \\
\hline \hline
\end{tabular}

\section{Conclusions}

The main discernible contribution of the paper is the correlation of the information provided from different validity indexes in order to define a determined number of clusters. Another contribution 
of the paper is the guidance search provided by the algorithm to select the optimal number of clusters in an unsupervised way. In principle, there is not a validity index that can be totally accurate, so redundant data of the partitions embedded in the validity measures will let us a better proposal of the optimal number of clusters of the image. Simulations and experiments are shown.

\section{REFERENCES}

[1] H.Farrakhbakht, A. Masoumzadeh Tork, E. Yazdan Talab, H. Ramezanpour, G. Darmani, An Adaptive Filter for Noise Cancelling in Mammography Images Based on Cellular Automata, International Review on Modelling and Simulations, vol. 5(3), pp. 1322-1326, June, 2012.

[2] G.M., Rajathi, R., Rangarajan, A New Dual Tree Wavelet Based Image Denoising Using Fuzzy Shrink and Lifting Scheme, International Review on Modelling and Simulations, vol. 6(2), pp. 13221326, December, 2013.

[3] D.F.T. Gamarra, L.K. Pinpin, C. Laschi, P. Dario Forward Models Applied in Visual Servoing for a Reaching in the iCub Humanoid Robot. In Applied Bionics and Biomechanics, vol. 6, pp. 345-354, 2009.

[4] D.F.T., Gamarra, Ballistic Reaching Using Visuomotor Maps in a Robot Manipulator for Starting a Visually Guided Task, International FLINS Conference on Decision Making and Soft Computing, 2014, João Pessoa, Brazil.

[5] D.F.T., Gamarra, T.F., Bastos, M.F., Sarcinelli, Validity Guided Controling of Navigation of a Mobile Robot in a corridor with Redundant Controllers, IEEE International Conference on Robotics and Automation (ICRA), 2005, Barcelona, Spain.

[6] L.Lucchese, S.K. Mitra, Color Image Segmentation: A state of the art Survey, Image Processing, Vision, and Pattern Recognition. In, Proceedings of the Indian National Science Academy (INSA-1), New Delhi, India, vol. 67A(2), pp. 207-221, 2001.

[7] N.V., Lopes, P.A.M. do Couto, H. Bustince: Automatic Histogram Threshold Using Fuzzy Measures. IEEE Transactions on Image Processing, vol 19 (1), pp. 199-204, 2010.

[8] H.D., Cheng, J.R., Chen, J. Li, Threshold Selection Based on Fuzzy c-Partition Entropy Approach. Pattern Recognition, vol 31 (7), pp. 857-870, 1998.

[9] D.F.T., Gamarra, Forward Models with Cluster Validity Criteria Applied in Ballistic Reaching for Visual Servoing, International Review on Modelling and Simulations, vol.(6), pp. 1939-1947, December, 2013.

[10] A.M. Bensaid, L.O., Hall, J.C., Bezdek, L.P., Clarke, M. L., Silbiger, J.A., Arrington, R. F., Murtagh, Validity-Guided ( $\mathrm{Re})$ Clustering with Applications to Image Segmentation. IEEE Transactions on Fuzzy Systems, vol. 4(Issue 2):112-123, May, 1996.

[11] R.P., Velthuizen, Validity Guided Clustering for Brain Tumor Segmentation, IEEE 17th Annual Conference Engineering in Medicine and Biology Society, pp. 413-414, 1995, Tampa, Florida, USA.

[12] Z., Wang, Comparison of Four Kinds of Fuzzy C-Means Clustering Methods. In: Symposium on Informatics Processing ISIP, pp. 563-566, 2010.

[13] M., Setnes, and R. Babusca, A Fuzzy Relational Classifier Trained by Fuzzy Clustering. IEEE Transactions on Systems, Man, and Cybernetics, Vol. 29(5), pp. 619-625, 1999.

[14] N., Jain, and S. Shukla Fuzzy Databses Using Extended Fuzzy C-Means Clustering. Journal of engineering Research and Applications, vol. 2(3), pp. 1444-1451, 2012.

[15] K. .M., Tarek, and B. Forouk, Kohonen Maps Combined to Fuzzy C-Means, a Two Level Clustering Approach. Application to Electricity Load Data. In Self Organizing Maps- Applications and Novel Algorithm Design, 2011.

[16] J.C., Bezdek Pattern Recognition with Fuzzy Objective Function Algorithms, Plenum, N.Y., 1981.

[17] X.L., Xie, and G.A. Beni, Validity measure for fuzzy clustering, in IEEE Transactions on Pattern Analysis and Machine Inteligence, 13(8), pp. 841-847, 1991.

[18] B.. Balasko, J. Abonyi, and B. Feil Fuzzy Clustering and Data Analysis Toolbox, http://www.abonyilab.com/software-and-data/fclusttoolbox, 2005.

[19] http://research.microsoft.com/en

us/um/cambridge/projects/visionimagevideoediting/segmentation/grabcut.htm. 
International Journal of Computer Science \& Information Technology (IJCSIT) Vol 7, No 3, June 2015

\section{Author}

Daniel Fernando Tello Gamarra was born in Peru in 1976, received his BSc. Degree in mechanical engineering from Universidad Nacional del Centro del Peru (UNCP), in Huancayo, Peru, in 1999, and his MSc in electrical Engineering from Universidade Federal do Espirito Santo (UFES), in Vitoria, Espirito Santo, Brazil, in 2004 and $\mathrm{PhD}$ degree in Biomedical Robotics from Scuola Superiore Santa Anna, Pisa, Italy, 2009. He is Professor at the Universidade Federal de Santa Maria (UFSM), in the Department of Control and Automation in Santa Maria, Rio Grande do Sul, Brazil. His current research interest centers on robotics, computational vision

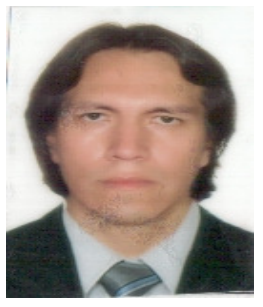
and machine learning. 